\title{
Patient Satisfaction as a Component of Health Care Quality in Otolaryngology
}

\author{
Cynthia Chen $\cdot$ Emily F. Boss
}

Published online: 18 February 2014

(C) Springer Science+Business Media New York 2014

\begin{abstract}
Reporting patient feedback and experience of care is a key component in the national effort to provide transparency and improve the overall quality of healthcare delivered in the US. With the passage of the Patient Protection and Affordable Care Act in 2010, Medicare reimbursements to medical organizations and individual providers are now being linked to process of care and outcomes, as well as experience measures, which include patient satisfaction scores. Despite the importance of gauging the patient experience to improve care and communication, it remains relatively unknown whether current methods of assessing patient satisfaction scores accurately reflect the patients' experiences, how they relate to patient health status, how patient cultural background and demographics affect reporting of experience, and how these scores are impacted by the variety of settings in which otolaryngology in practiced. Importantly, whether or not increased patient satisfaction is correlated with improved patient health and a decrease in healthcare costs remains to be seen. The significance of patient satisfaction within the discipline of surgery, more specifically otolaryngology, has not been clearly elucidated. In this report, we review recent research surrounding the measurement and reporting of patient satisfaction in otolaryngology.
\end{abstract}

Keywords Quality and outcomes · Patient satisfaction · Health care - Otolaryngology

C. Chen $(\bowtie) \cdot$ E. F. Boss

Department of Otolaryngology - Head and Neck Surgery, Johns Hopkins University School of Medicine, Baltimore, MD, USA e-mail: cchen185@jhmi.edu

\section{Introduction}

Improving the quality of healthcare in the US while curbing the soaring cost has become a national priority. The Institute of Medicine (IOM) [1] has stated plainly that "Medicare is not getting the best value for the services it purchases" despite the enormous amount of money invested in the health of Americans. In their seminal report Crossing the Quality Chasm, the IOM defined patientcenteredness as one of six required components for the provision of quality healthcare, along with safety, timeliness, effectiveness, efficiency, and equity [2]. Furthermore, experts propose that when considering quality, "patients' experiences should be the fundamental source of the definition" [3].

The emphasis placed on patient satisfaction as a focal measure is now propelling the development of pay-forperformance types of reimbursement formulas that link patient satisfaction scores directly to monetary reimbursement. This trend is impacting health systems, hospitals, employers, and individual providers. With the implementation of the Patient Protection and Affordable Care Act (PPACA), further payment reform proposed by the Centers for Medicare and Medicaid Services (CMS) will be going into effect. These changes range from how Medicare reimburses hospitals and physicians to how physicians will maintain their certifications.

Clinician-patient interaction scores may constitute up to $30 \%$ of the quality-value formula in Medicare payment reform programs such as Accountable Care Organizations [4]. Many large institutions will be incorporating patient satisfaction scores into determining qualification for financial incentives or penalties for physicians. Specialty boards, including the American Board of Otolaryngology, will incorporate objective measures of patient experience 
as a component of part IV [5] of the requirement for maintenance of board certification for physicians. As health industries transition emphasis from "disease-centered" (or "doctor-centered") to patient-centered environments, organizations are evolving to appeal to the patient as a consumer in the healthcare marketplace.

National and state initiatives are in place to evaluate physicians based on measures that include patient satisfaction. Reporting of inpatient Consumer Assessment of Healthcare Providers and Systems (CAHPS) survey scores, developed by the Agency for Healthcare Quality and Research, is required for hospitals that accept Medicare [6]. Following suit with the inpatient sector, a PPACA clause mandates CMS to expand public reporting of patient satisfaction to the outpatient, provider-level realm. The Clinician and Group CAHPS (CG-CAHPS) and Surgical CAHPS surveys have been developed to help providers meet this mandate [7] (see Table 1). In an effort to provide the patient with real-time comparison data on their doctors, these scores are anticipated to be reported on the "Physician Compare" website [8] managed by CMS, although the public reporting function of this website is still in development [9]. Because these surveys are in different stages of validation and standardization for various patient groups, many institutions contract for-profit vendors to collect and report patient experience data for inpatient and provider-specific practices. There are also various public open-source websites fully devoted to patient experience and subjective review of physicians that are peer-reviewed only.

The impetus for improved transparency and reporting of patient satisfaction stems from the drive to improve quality of care. However, because of the inherent nature of a medical system that is organized into subspecialties, it is difficult to standardize the experience of one patient to that of another. We seek here to provide a review in terms of understanding how patient experience scores apply to the setting of otolaryngology practice.

\section{The Relationship Among Patient Experience, Physical Health, and Demographics is Uncertain}

Due to the energy and resources surrounding patient experience measurement and reporting, understanding how these scores mesh with clinical practice is paramount. The relationship between patient satisfaction and inpatient care has been recently explored; however, there is no clear conclusion that higher patient satisfaction is indeed congruent with higher quality of care or lower cost and utilization [10॰]. Fenton et al. evaluated a nationally representative sample of more than 50,000 adult respondents to the Medical Expenditure Panel Survey (MEPS), which included patient-reported satisfaction scores from the inpatient H-CAHPS. Higher patient satisfaction was associated with greater use of inpatient care, higher healthcare utilization, and prescription drug use, as well as increased mortality [11•]. A separate analysis of 2003 MEPS data by Fu and Wang [12] showed no significant relationship between patient satisfaction and healthcare expenditures. Furthermore, the Dartmouth Atlas Project has shown that high inpatient care intensity, as measured by greater resource utilization, has been shown to have negative or no correlation with patient satisfaction and perception of quality of care [13].

Even less is known about clinical predictors of patient satisfaction scores in outpatient practice and the effect of care acuity. Chang et al. investigated CAHPS data from geriatric patients from two managed care organizations and looked for correlation of patient satisfaction ratings and technical quality of care, which was scored based on care processes and state of patient health. They found that better communication was associated with high global ratings of health care in this cohort of elderly patients. Technical quality of care, defined as delivery of appropriate care for specific medical problems, was not found to be significantly correlated with global subjective ratings of care [14]. Patient expectations for diagnostic testing and treatment may also influence physician behaviors and satisfaction scores in the outpatient setting $[15,16]$. In a study by Macfarlane et al. [17], patient pressures and expectations were shown to persuade physicians to prescribe antibiotics, even when not definitely indicated, and patients who thought they had an infection but did not receive an antibiotic prescription were dissatisfied with care. The impact of health factors including health status or ordering of prescriptions, tests, or recommendation for surgery on patient satisfaction has not been studied within otolaryngology.

Assessing patient satisfaction in the pediatric population becomes more complex for several reasons. Surveys reflect parental experience and concerns as they act as proxy for their children. The clinician must interact and communicate with multiple members of family and the child. Time at a physician's office or hospital means time off from both parental and child obligations such as work and school. There are many other factors such as these that make the experience of a child different than that of an adult. Because many otolaryngologists commonly treat children, understanding the differences and challenges in patient experience measurement across patient ages is important for our specialty.

There has been a great deal of research into patient experience as a measure of care quality in the pediatric population. Homer et al. reviewed Picker/Commonwealth Patient Centered Care survey responses obtained from parents of children who received inpatient care at a tertiary academic hospital over 4 years. Parents most commonly 
reported problems regarding hospital discharge planning and pain management. Questions relating to communication between providers and parents correlated most strongly with overall quality. The least number of complaints were made about communication about surgery and transmission of information to children. The authors also found parents who reported more problems and rated pediatric care lower were parents who rated their child's overall health status as lower. Additionally, parents of economically disadvantaged and minority children rated care lower [18].

Several studies have compared adult and pediatric care assessments, and the results are contradictory. Chong et al. [19] reviewed their data from CAHPS surveys conducted in Iowa, and found that timeliness of appointment and short waiting-room time were more important to adults, whereas courtesy and helpfulness of staff were more important to parents of children. Zhan et al. [20] also compared CAHPS data between adult and pediatric patients, and found that adults rated their care higher than parents of pediatric patients. Boss and Thompson had similar findings in their comparison of adult and pediatric outpatient Press Ganey survey scores. Chen et al. compared CAHPS data between adults and children, and between a West Coast health system to one in the Midwest. In this study, the authors found that care of children was viewed more positively than that of adults. There were differences between the two regions in the country, with adult patients from the Western US perceiving office staff courtesy and helpfulness more positively than pediatric patients. There were more positive provider-related scores from pediatric patients in areas such as coordination of care and overall rating of the provider. In the Midwest, pediatric surveys were more positive than for adults in areas such as access to care, provider communication, and office staff courtesy and helpfulness [21]. The majority of these studies evaluated patient experience in the primary care setting.

Preliminary studies of data from different surveys across various healthcare settings show the wide variety of patient experience surveys and how difficult it is to make broad generalizations about how patients perceive their medical care. Currently, it is difficult to say whether patients who report the highest satisfaction have better clinical outcomes or utilize the medical system more efficiently.
Table 1 Sample medley of questions related to provider care asked on patient experience surveys

From [28-30], with permission
Consumer Assessment of Healthcare Providers and Systems (CAHPS)-Surgical Care Survey (Agency for Healthcare Research and Quality)

A health provider could be a doctor, nurse, or anyone else you would see for health care. Before your surgery, did anyone in this surgeon's office give you all the information you needed about your surgery?

During your office visits before your surgery, did this surgeon ask which way to treat your condition you thought was best for you?

Before you left the hospital or surgical facility, did this surgeon discuss the outcome of your surgery with you?

Did anyone in this surgeon's office explain what to expect during your recovery period?

Did this surgeon make sure you were physically comfortable or had enough pain relief after you left the hospital or surgical facility where you had your surgery?

Using any number from 0 to 10 , where 0 is the worst surgeon possible and 10 is the best surgeon possible, what number would you use to rate all your care from this surgeon?

Press Ganey ${ }^{\circledR}$ Associates Medical Practice Survey

Questions asking the patient to rate as very poor, poor, fair, good, very good:

Friendliness/courtesy of that provider

Concern of the care provider for your questions and worries

Your confidence in this care provider

Likelihood of your recommending this care provider to others

Speed of the registration process

NRC Picker (National Research Corporation)

Were you able to get an appointment as soon as you wanted it?

If you had to leave a message for the clinic, was your call returned within one business day?

How would you rate the courtesy of the person who made your appointment?

If your child's appointment did not begin on time, did someone give you a reason for the delay?

Did your child's health care provider treat you with respect and dignity?

Did you child's health care provider help you do a good job caring for your child?

Do you have confidence and trust in the care provider treating your child? 


\section{Patient-Centered Care in Otolaryngology}

There has been active interest in learning about patient outcomes and satisfaction in the context of otolaryngology. Otolaryngic procedures are performed in various settings (academic tertiary care centers, community hospitals, outpatient surgical centers), and many studies have investigated patient safety throughout the spectrum of settings [22, 23]. However, it is still rather unknown whether otolaryngology as a specialty has adopted a truly "patient-centered" model. For example, otolaryngology is still heavily entrenched, like other surgical specialties, within a traditional fee-for-service paradigm. Whether the patient-centered, value-based, qualityrewarding pay-for-performance will be well received within the practice of Otolaryngology is still largely unknown.

An analysis by Boss and Thompson of Press Ganey Medical Practice surveys within otolaryngology comparing outpatient experience in academic institutions to nonacademic settings showed that overall patient satisfaction scores were lower from academic outpatient settings compared to those from non-teaching, community settings. The most negative scores were related to factors such as wait time, speed of registration, and comfort of the waiting room. However, scores related to loyalty, likelihood to recommend the practice, and satisfaction with the care provider were higher from academic clinics [24•]. While factors related to the surgeon were most highly rated, the "promptness in returning your phone call" negatively correlated to practice loyalty.

These findings reflect several issues that affect the flexibility of academic institutions to comply with the call to transition from provider-centered to patient-entered care. Resident training is often a main pillar of the mission of a teaching hospital, and involvement of residents in patient care often adds additional time to a clinic visit and sometimes complicates communication. Teaching institutions tend to serve more socioeconomically and culturally diverse patient populations, who may have differing needs, values, or expectations of their healthcare experiences. There may be less funding available for public space improvements and care-coordination programs in academic facilities. Finally, teaching institutions are dedicated to research, a focus that could potentially alter the outlook of physicians and may affect availability of timely appointments. However, the authors point out that customer loyalty, in addition to increased revenues and productivity, and employee satisfaction are pillars of successful business models in the retail sector, and there is room for healthcare institutions, academic or community, to follow suit.

\section{Family Satisfaction in Pediatric Otolaryngology}

Measuring patient satisfaction in pediatric otolaryngology adds a layer of complexity for several reasons. Children constitute a significant proportion of the patients cared for by otolaryngologists. But, as mentioned before, what separates the child from the self-sufficient adult patient is the family context that must be considered and included in the care of the patient. Surveys assessing patient experience typically reflect parental experience with care since the patient is typically represented by parents/caregivers. Socioeconomic and cultural factors play heavily into expectations when it comes to the care of children, and it is unknown how differing levels of education and literacy affect the ability of parents to communicate about their experiences.

Zopf et al. [25] surveyed patients and families at an academic pediatric otolaryngology clinic for 1 year and found that there was a significant relationship between patient satisfaction and satisfaction with the provider, examination room wait times, and reception area wait times. This study gave insight into what matters to parents: time spent waiting and direct interaction with the provider.

In a study examining national Press Ganey Medical Practice Survey scores to determine satisfaction scores from pediatric otolaryngology outpatient practices compared to those from adult patients, Boss and Thompson found that mean scores were lower and different for children across all survey domains except for care provider where the difference was not significant. Within the pediatric group, scores were lowest for children between 0 and 6 years old compared to those aged 6-12 and 12-18. These scores across all service domains typically increased with age into adulthood. This study also demonstrated that the perceived parent experiences of care are different from those of adults, and assessment of patient experience should be conducted with these differences in mind [26].

Nieman et al. examined Press Ganey Medical Practice Survey scores within a pediatric division at an urban tertiary academic institution in order to elucidate how social demographics such as race and insurance status were related to disparities in parent participation with pediatric otolaryngology satisfaction surveys. They found that overall white families and privately insured families were much more likely to respond to the surveys. In this practice, the authors note that the response rate to patient satisfaction surveys was not representative of the culturally and socioeconomically diverse population served in the clinic. Rates of nonresponse have also been noted to be higher in patients with less favorable views of their care. Indeed, cultural competence and health literacy are other factors to consider in order to effectively evaluate the otolaryngology patient experience [27].

\section{Conclusion}

As we enter a new era of measuring quality and increasing transparency, it is imperative to understand how the 
medical community gathers data, accounts for variation or diversity of the patient population, and how we interpret and apply patient satisfaction scores to our practices and reimbursement.

\section{Compliance with Ethics Guidelines}

Conflict of Interest Cynthia Chen and Emily F. Boss declare that they have no conflicts of interest.

Human and Animal Rights and Informed Consent This article does not contain any studies with human or animal subjects performed by the authors.

\section{References}

Papers of particular interest, published recently, have been highlighted as:

- Of importance

1. Institute of Medicine. Rewarding provider performance: aligning incentives in medicare. Washington, DC: National Academies Press; 2006.

2. Institute of Medicine. Crossing the quality chasm: a new health system for the 21st century. Washington, DC: National Academies Press; 2001.

3. Berwick DM. A user's manual for the IOMs "Quality Chasm" report. Health Aff (Millwood). 2002;21:80-90.

4. Centers for Medicare and Medicaid Services. Guide to quality performance standards for accountable care organizations starting in 2012: pay for reporting and pay for performance. http://www. cms.gov/Medicare/Medicare-Fee-for-Service-Payment/sharedsavings program/Downloads/ACO-Guide-Quality-Performance-2012.PDF.

5. American Board of Otolaryngology. http://www.aboto.org/part4. html.

6. Agency for Healthcare Research and Quality. https://cahps.ahrq. gov/surveys-guidance/docs/sample_tele_script_surgical_care_ surveyx.docx.

7. Davis PA, Hahn J, Morgan PC, Stone J, Tilson S. Medcaire provisions in the patient protection and affordable care act (PPACA). 2010.

8. Medicare.gov. Agency for Healthcare research and quality, US Dept of Health and Human Services. Consumer assessment of healthcare providers and systems. http://www.medicare.gov/physi ciancompare/search.html.

9. US Department of Health and Human Services. http://www. hospitalcompare.hhs.gov/. 2001.

10. - Manary MP, Boulding W, Staelin R, Glickman SW. The patient experience and health outcomes. N Eng J Med. 2013;368(3): 201-3.

11. - Fenton JJ, Jerant AF, Bertakis KD, Franks P. The cost of satisfaction: a national study of patient satisfaction, health care utilization, expenditures, and mortality. Arch Intern Med. 2012;172(5): 405-11. This paper discusses analysis of national data that shows that higher patient satisfaction scores do not necessarily correlate with better patient outcomes and more efficient usage of healthcare resources.
12. Fu AZ, Wang N. Healthcare expenditures and patient satisfaction: cost and quality from the consumer's perspective in the US. Curr Med Res Opin. 2008;24(5):1385-94.

13. Wennberg JE, Bronner K, Skinner JS, Fisher ES, Goodman DC. Inpatient care intensity and patients' ratings of their hospital experiences. Health Aff (Millwood). 2009;28(1):103-12.

14. Chang JT, Hays RD, Shekelle PG, MacLean CH, Solomon DH, Reuben DB, Roth CP, Kamberg CJ, Adams J, Young RT, Wenger NS. Patients' global ratings of their health care are not associated with the technical quality of their care. Ann Intern Med. 2006; 144:655-72.

15. Kravitz RL, Bell RA, Azari R, Kelly-Reif S, Krupat E, Thom DH. Direct observation of requests for clinical services in office practice: what do patients want and do they get it? Arch Intern Med. 2003;163(14):1673-81.

16. Lin CT, Albertson GA, Schilling LM, et al. Is patients' perception of time spent with the physician a determinant of ambulatory patient satisfaction? Arch Intern Med. 2001;161(11):1437-42.

17. Macfarlane J, Holmes W, Macfarlane R, Britten N. Influence of patients' expectations on antibiotic management of acute lower respiratory tract illness in general practice: questionnaire study. BMJ. 1997;315(7117):1211-4.

18. Homer CJ, Marino B, Clear PD, Alpert HR, Smith B, Crowly Ganser CM, Brustowicz RM, Goldmann DA. Quality of care at a children's hospital. Arch Pediatr Adolesc Med. 1999;153:1123-9.

19. Chong K, Damiano PC, Belin TR, et al. Psychometric performance of the Consumer Assessment of Healthcare Providers and Systems (CAHPS) 4.0 adult health plan survey. Prim Health Care: Open Access (submitted).

20. Zhan C, Sangl J, Meyer GS, et al. Consumer assessments of care for children and adults in health plans: how do they compare? Med Care. 2002;40:145-54.

21. Chen AY, Elliott MN, Spritzer KL, Brown JA, Skootsky SA, Rowley C, Hays RD. Differences in CAHPS reports and ratings of health care provided to adults and children. Med Care. 2012; 50:S35-9.

22. Grisel J, Arjmand E. Comparing quality at an ambulatory surgery center and a hospital-based facility: preliminary findings. Otolaryngol Head Neck Surg. 2009;141:701-9.

23. Shah RK, Welborn L, Ashktorab S, Stringer E, Zalzal GH. Safety and outcomes of outpatient pediatric otolaryngology procedures at an ambulatory surgery center. Laryngoscope. 2008;118:1937-40.

24. - Boss EF, Thompson RE. Patient satisfaction in otolaryngology: can academic institutions compete? Laryngoscope. 2012;122: 1000-09. Important published report of analysis of national patient satisfaction scores obtained from ambulatory clinics in Otolaryngology, comparing the academic and community setting.

25. Zopf D, Joseph AW, Thorne MC. Patient and family satisfaction in a pediatric otolaryngology clinic. Int J Pediatr Otorhinolaryngol. 2012;76:1339-42.

26. Boss EF, Thompson RE. Patient experience in outpatient pediatric otolaryngology. Laryngoscope. 2012;122:2304-10.

27. Nieman CL, Benke JR, Ishman SL, Smith DF, Boss EF. Whose experience is measured? A pilot study of patient satisfaction demographics in pediatric otolaryngology. Laryngoscope. 2014; 124:290-4.

28. Agency for Healthcare Research and Quality, US Department of Health and Human Services. https://cahps.ahrq.gov.

29. Press Ganey ${ }^{\circledR}$ Associates Medical Practice. http://www.pressganey. com/index.aspx.

30. National Research Corporation. http://www.nationalresearch. com. 